\title{
A clinical study of ectopic pregnancy: a five-year institutional experience
}

\section{Malavika J. C.*, Prema Prabhudev, Bandamma N. S.}

Department of Obstetrics and Gynecology, SSIMS and RC, Davangere, Karnataka, India

Received: 11 May 2017

Accepted: 16 May 2017

\section{*Correspondence:}

Dr. Malavika J. C.,

E-mail: malavika.jcm@gmail.com

Copyright: () the author(s), publisher and licensee Medip Academy. This is an open-access article distributed under the terms of the Creative Commons Attribution Non-Commercial License, which permits unrestricted non-commercial use, distribution, and reproduction in any medium, provided the original work is properly cited.

\begin{abstract}
Background: Ectopic pregnancy contributes significantly to maternal morbidity and mortality. There is a steady increase in the incidence of ectopic pregnancy over the past few decades, owing to rise in sexually transmitted infections, subfertility and its treatment with ART techniques. Objective of present work was to study the rate of ectopic pregnancy, associated risk factors, clinical course and management at SSIMS and RC.

Methods: This is a retrospective study carried out at SSIMS and RC, Davangere from 01 $1^{\text {st }}$ January 2012 to $30^{\text {th }}$ April 2017. A detailed analysis of case sheets done and all parameters analyzed. Institutional ectopic rate was calculated per 1000 deliveries.

Results: Out of 43 women with ectopic gestation, $40(93.02 \%)$ were tubal ectopic and $3(6.98 \%)$ were ovarian ectopic. Institutional rate of ectopic is 11.54 per 1000 deliveries. STI's are the commonest risk factor followed by subfertility. Diagnostic aid commonly used was pelvic ultrasound. There were no maternal deaths due to ectopic pregnancy. One third of the women presented with shock. 52.5\% tubal and 66.6\% ovarian ectopics were ruptured. All women with tubal ectopic were managed by salpingectomy either by open or laparoscopic method and ovarian ectopics managed by ipsilateral oophorectomy. Two-thirds of the women received blood transfusion and 5-7 days were required for post-operative recovery.

Conclusions: Ectopic pregnancy is one of the commonest gynecological emergency and there is a yearly increase in the rate of ectopic. STI's and subfertility are important risk factors. Prevention, early detection and treatment of STI's may play an important role in reducing the rate of ectopic pregnancy.
\end{abstract}

Keywords: Ectopic pregnancy, Pelvic ultrasound, STI's, Subfertility

\section{INTRODUCTION}

Ectopic pregnancy is implantation of the fertilized ovum outside the uterine cavity. Depending on the location it can be tubal, ovarian, cervical, abdominal and can even occur on the caesarean scar. ${ }^{1}$ Although the total number of pregnancies are decreasing with time there is an increase in the incidence of ectopic gestation over the past few decades, owing to rise in sexually transmitted infections, increasing subfertility and its treatment with ART techniques, and many other risk factors. Tubal ectopics comprise the majority accounting for almost 95 to $97 \%$ of total ectopics. Tubal ectopics are usually associated with predisposing risk factors which are known to damage the tubal epithelium. ${ }^{1,2}$

Most common risk factor is sexually transmitted infections and PID resulting in salpingitis and partial occlusion of fallopian tube. With the advent of antibiotic treatment for PID and STI's, agglutination of cilia can occur and result in band formation and partial tubal occlusion. Sexually transmitted infections (particularly 
chlamydia trachomatis) are associated with subclinical damage to the tubal epithelium contributing to ectopic pregnancy. IUCD's although associated with very low failure rate, when pregnancy occurs with IUCD in situ, it is more often ectopic. ${ }^{2-4}$ Other risk factors for tubal ectopic include previous history of tubal surgery (tubal ligation, tubal reconstrctive surgery), past history of tubal ectopic, increasing use of ART techniques (incidence of heterotopic pregnancy is also high), developmental anomaly of fallopian tube (intramural polyp, tubal diverticula etc) and women exposed to diethyl- stilbestrol in utero. Other causal factors include multiple sexual partners, early sexual debut, vaginal douching and cigarette smoking. Infertility itself is a risk factor for tubal ectopic associated with almost two-fold increased incidence, although there may be no evident tubal abnormality. ${ }^{4}$ Ectopic pregnancy occuring outside the fallopian tube are usually not associated with these predisposing risk factors.

Ovarian ectopics are rare and comprise 3.2 to $3.5 \%$ of ectopic pregnancies. The diagnosis is often surgical and requires histo-pathological confirmation as it is difficult to differentiate from hemorrhagic corpus luteum intraoperatively. ${ }^{2,3}$

Clinical trial of an extra-uterine pregnancy is pain, uterine bleeding and an adnexal mass. Earlier the presentation, less severe and less predictive are the clinical manifestations. Commonly used diagnostic modality is transvaginal ultrasound which allows a detailed assessment of uterus, adnexa and presence or absence of free peritoneal fluid. ${ }^{2,3}$ Visualization of a complex adnexal mass in combination with an empty uterus has a sensitivity of $97 \%$, specificity of $99 \%$ and a positive and negative predictive value of $98 \%$, provided serum beta-hCG concentrations exceed 1,000 IU/L. Serum beta hCG assay aid the diagnosis and management of ectopic pregnancy. Serum progesterone assay may also be used to differentiate an extrauterine pregnany from a non-viable intrauterine pregnancy. Culdocentesis is one of the diagnostic modality for identifying the presence of intraperitoneal bleeding. Although invasive, laparoscopy is considered gold standard in the detection of ectopic pregnancy when the dilemma is not resolved by noninvasive techniques. ${ }^{2,3}$

The most common treatment modality opted for in a case of ectopic gestation is surgical unless they fulfill the criteria for medical management with Methotrexate. Total salpingectomy on the affected side is the preferred surgical procedure in case of tubal ectopic gestation. Partial salpingectomy and conservative surgical procedure such as linear salpingotomy, salpingostomy are rarely performed these days owing to increased failure rate of the procedure and also recurrence of ectopic pregnancy at a later date in the same site. Choice between open and laparoscopic salpingectomy depends on clinical scenario, amount of hemoperitoneum, facilities available and technical skills of the operating surgeon.,
This study aims to provide a detailed insight into the incidence, clinical manifestations, associated risk factors, diagnostic modality, treatment methods and complications of ectopic pregnancy in women presenting to SSIMS and RC, a tertiary referral hospital in Davangere.

\section{METHODS}

This is a retrospective study carried out at the department of Obstetrics and Gynecology at S.S Institute of Medical Sciences and Research Centre, Davangere, from $01^{\text {st }}$ January 2012 to $30^{\text {th }}$ April 2017. The operating theatre register and gynec ward register were carefully studied to identify the cases admitted and operated for ectopic gestation. Case sheets of subjects who were suspected of ectopic gestation were obtained from medical records department and detailed analysis of various parameters done. Information collected included variables like age, area of residence, marital status, obstetric score, predisposing factors, clinical presentation, investigations done, surgery performed, intra-op findings, complications, duration of post op hospital stay, blood transfusion and complications.

The data collected were entered on an excel spreadsheet. The labour ward register was used to collect the details of number of deliveries during the study period. Data analysis performed using excel spreadsheet and expressed in simple percentages. Institutional ectopic rate was calculated per 1000 deliveries. Number of cases referred from different centres were noted.

\section{RESULTS}

A total of 46 suspected cases of ectopic pregnancy were included in the study period based on clinical presentation and diagnostic studies. Out of 46 women suspected of ectopic pregnancy, 43 women were found to have ectopic pregnancy and remaining 3 women were not found to have ectopic pregnancy on performing diagnostic laparoscopy.

Out of the 3 women who were not found to have ectopic gestation, 1 of them had corpus luteal cyst hemorrhage mimicking ectopic pregnancy and other 2 had no evidence of ectopic gestation on diagnostic laparoscopy and check curettage was done in both the cases and were subsequently found to have failing intrauterine pregnancy on histopathological examination.

Out of 43 women with ectopic gestation, 40 were tubal ectopic pregnancy accounting for $93.02 \%$ of cases and 3 were ovarian ectopic accounting for $6.98 \%$ of cases. The institutional rate of ectopic pregnancy was calculated per 1000 deliveries. The overall institutional rate is $11.54 \mathrm{per}$ 1000 deliveries.

The yearly institutional rate is calculated and is depicted in Table 1. The rate of ectopic calculated for the year 
2017 includes only 4 months from January to April. There was no mortality recorded in women with proven ectopic pregnancy. The rate of ectopic is seen to gradually rise especially so in first 4 months of 2017 approximately accounting for 35.33 ectopics per 1000 deliveries.

Table 1: Yearly rate of ectopic at SSIMS and RC.

\begin{tabular}{|llllllll|}
\hline Year & 2012 & 2013 & 2014 & 2015 & 2016 & $2017 *$ & Total \\
\hline No. of ectopics & 4 & 3 & 7 & 9 & 10 & 10 & 43 \\
\hline Total no. of deliveries & 449 & 575 & 631 & 776 & 1010 & 283 & 3724 \\
\hline Rate of ectopic & 8.9 & 5.2 & 11.09 & 11.59 & 9.9 & 35.33 & 11.54 \\
\hline
\end{tabular}

*rate is calculated only for first four months of the year 2017

\section{Demographic details}

Majority of the women belonged to age 20-24 and 25-29 years age group. Age preponderance is depicted in Table 2. Mean age for women with ectopic pregnancy is $25.2 \pm 2.6$ years. Majority of the women in the study carried out were married and only 2 were unmarried. 20 women in study were urban dwellers and 23 belonged to rural area. The data regarding education and employment of the women in study were poorly documented. Out of 43 women with ectopic pregnancy, 19 (44.18\%) were nulliparous, $12(27.9 \%)$ were para $1,11(25.58 \%)$ were para 2 and $1(2.32 \%)$ was para 4 . The median parity was 2.

Table 2: Age preponderance in women with ectopic pregnancy.

\begin{tabular}{|lll|}
\hline Age group (years) & No. of women & $\%$ of women \\
\hline $20-24$ & 17 & 39.53 \\
\hline $25-29$ & 17 & 39.53 \\
\hline $30-34$ & 7 & 16.27 \\
\hline $35-39$ & 2 & 4.65 \\
\hline Total & 43 & 100 \\
\hline
\end{tabular}

\section{Predisposing factors}

A total of $13(30.23 \%)$ women in study had subfertility and out of them $5(11.62 \%)$ were found to have primary subfertility and $8(18.6 \%)$ women had history of secondary subfertility. History of pelvic inflammatory disease was documented in $5(11.62 \%)$ women and sexually transmitted infections were documented in 15 (34.88\%) women. Past history of ectopic was recorded in $3(6.97 \%)$ women and $10(23.2 \%)$ women in study previously suffered from abortions/miscarriages. Prior use of intrauterine contraceptive device (IUCD) was documented in $2(4.65 \%)$ cases. In the study group only $1(2.32 \%)$ woman was a smoker. History of ovulation induction was documented in $4(9.32 \%)$ women, history of usage of injection depot medroxy progesterone was noted in $3(6.97 \%)$ women. Past history of tubectomy noted in $2(4.65 \%)$ women, emergency contraception was used by $1(2.32 \%)$ woman, MTP kit was used by 2
(4.65\%) women. Medical history of hypothyroidism was documented in $3(6.97 \%)$ women.

\section{Presenting symptoms}

Abdominal pain was the most common presenting symptom and it was documented in 39(90.69\%) women. The next most common complaint was amenorrhea noted in $32(74.41 \%)$ women and abnormal vaginal bleeding was noted in $28(65.11 \%)$ women. $10(23.2 \%)$ women complained of syncope and $4(9.32 \%)$ women presented with urinary retention. $11(25.58 \%)$ women presented with shock. (Shock is defined as heart rate more than 90 beats per minute and blood pressure less than 90/60 $\mathrm{mmHg}$ ). Clinical symptoms and signs are tabulated in Table 3 .

Table 3: Clinical symptoms and signs in women with ectopic pregnancy.

\begin{tabular}{|l|l|}
\hline Symptoms and signs & No. of women \\
\hline Symptoms & \\
\hline Abdominal pain & 39 \\
\hline Abnormal uterine bleeding & 28 \\
\hline Amenorrhea & 32 \\
\hline Syncope & 10 \\
\hline Urinary retention & 4 \\
\hline Vomiting & 4 \\
\hline Constipation & 1 \\
\hline Diarrhea & 1 \\
\hline Signs & \\
\hline Pallor & 27 \\
\hline Abdominal distension & 15 \\
\hline Lower abdominal tenderness & 27 \\
\hline Guarding & 10 \\
\hline Rigidity & 10 \\
\hline Abdominal mass & 8 \\
\hline POD bulge & 5 \\
\hline Cervical motion tenderness & 11 \\
\hline Adnexal mass & 32 \\
\hline Adnexal tenderness & 34 \\
\hline Shock & 11 \\
\hline Fever & 2 \\
\hline
\end{tabular}


Pre-operative hemoglobin ranged from 2 to $11.4 \mathrm{~g} / \mathrm{dl}$. Mean hemoglobin was $7.8 \pm 1.3 \mathrm{~g} / \mathrm{dl}$ and median hemoglobin was $8 \mathrm{~g} / \mathrm{dl}$. $\mathrm{Hb} 7$ to $10.9 \mathrm{~g} / \mathrm{dl}$ is categorized as mild anemia, 4-6.9 $\mathrm{g} / \mathrm{dl}$ is categorized as moderate anemia and $\mathrm{Hb}<4 \mathrm{~g} / \mathrm{dl}$ is categorized as severe anemia. In the study group, a total of $26(60.46 \%)$ women were anemic and out of them $16(37.2 \%)$ women had mild anemia, $8(18.6 \%)$ women had moderate anemia and 2 $(4.65 \%)$ women presented with severe anemia.

Diagnosis was confirmed mainly by pelvic ultrasound in all cases and only in $11(25.58 \%)$ cases serum beta-hCG was done to aid the diagnosis. In 5 (11.62\%) cases culdocentesis was done as an ancilliary diagnostic modality. The most common ultrasound finding was complex adnexal mass which was found in $22(51.16 \%)$ cases.

\section{Management}

All women with ectopic pregnancy were subjected for surgical line of management. Women were subjected to laparotomy or laparoscopic procedure depending on the hemodynamic status. Women who were hemodynamically stable were subjected for laparoscopic procedure. In our study, $29(67.44 \%)$ women underwent laparotomy and $14(32.55 \%)$ women underwent laparoscopic procedure. Out of 43 women, 35 (81.39\%) women were subjected for emergency surgery and 8 $(18.6 \%)$ women were operated upon electively as they were hemodynamically stable. In our study, 32 (74.41\%) women were subjected to general anesthesia and spinal anesthesia was given in $11(25.58 \%)$ women. General anesthesia was preferred in all emergency cases and spinal anesthesia was given in elective unruptured ectopic cases.

All tubal ectopics were managed by total salpingectomy and $5(11.62 \%)$ cases with tubal ectopic underwent concurrent tubal sterilization of the opposite fallopian tube as they did not desire future fertility. 3 cases of ovarian ectopic were managed by ipsilateral oophorectomy, 3 cases of interstitial ectopics were managed by total salpingectomy and partial myomectomy of the cornual end of the uterus. None of the cases had intra-operative complications.

\section{Intra-operative findings}

Out of 43 women with confirmed ectopic pregnancy, 40 (93.02\%) women had tubal ectopic, 3 (6.97\%) had ovarian ectopic. Out of 40 women with tubal ectopic, 14 $(32.55 \%)$ were right sided and $26(60.46 \%)$ were left sided. Among them, $12(27.9 \%)$ were located in isthmic region, 21 (48.83\%) in ampullary, 3 (6.97\%) were isthmo- ampullary, $2(4.65 \%)$ were fimbrial and 2 $(4.65 \%)$ were interstitial in location. Out of 40 tubal ectopics, $21(52.5 \%)$ tubal ectopics were ruptured and $19(47.5 \%)$ were unruptured. All 3 ovarian ectopics were right sided and $2(66.6 \%)$ ovarian ectopics were ruptured.
Macroscopic evidence of pelvic infection such as adhesions, hydrosalphinx, tubercles were noted in 9 $(20.93 \%)$ women with ectopic gestation. Evidence of endometriosis was noted in $1(2.32 \%)$ woman. Intraperitoneal bleed was documented in $35(81.39 \%)$ cases with an average blood loss of 700 to $1500 \mathrm{ml}$ (range being $200 \mathrm{ml}$ to 3 litres).

\section{Post-operative period}

A total of $33(76.74 \%)$ women received blood transfusion owing pre-operative anaemic status and intra-peritoneal bleed and out of them $11(33.33 \%)$ women had received intra-operative blood transfusion. All patients received peri-op and post-op broad spectrum antibiotics. Injectable iron was given to 5 women post-operatively to treat anaemic status. Average duration of post-op hospital stay was 7 days. $10(23.2 \%)$ women had delay in discharge from hospital due to anaemic status. The number of days of delay in such cases was 3 to 4 days compared to other cases. Contraceptive counselling was done in those women with intact opposite fallopian tube.

\section{DISCUSSION}

Ectopic pregnancy is one of the common gynaecological emergencies at SSIMS and R, C a tertiary referral hospital located in Davangere district of Karnataka. Although there was no mortality recorded in women with ectopic pregnancies, morbidity was high as more than half of the women with ectopic presented with ruptured ectopic gestation leading to hemodynamic compromise and one third of the women presented with hypovolemic shock on admission, more than two-thirds of the women had significant intra-peritoneal bleeding and around twothirds of the women had received blood transfusion periop and post-operatively. All women with tubal ectopic were managed with salpingectomy either open method or by laparoscopic approach and women with ovarian ectopic were managed with ipsilateral oophorectomy. The average duration of stay in the hospital was 5-7 days and it was prolonged to 10 days in $25 \%$ of the women as they were moderate to severely anaemic at the time of presentation and hence requiring a longer time for recovery. No intra-op and post-op complications were documented. Women referred from rural areas were more likely to present with ruptured ectopic pregnancy implying the need to intervene and detect ectopic pregnancy at earlier gestation.

The overall rate of ectopic at SSIMS and RC was 11.54 per 1000 deliveries. The rate of ectopic gestation has seemed to be on a rise every year and there is almost 3-4fold rise in ectopic pregnancies in the first four months $\left(01^{\text {st }}\right.$ January-30 ${ }^{\text {th }}$ April) of the year 2017. The rate of ectopic for the year 2017 was roughly calculated and it may not reflect the same rate at the end of the year 2017, yet there is a significant rise in the rate of the ectopic gestation. The rate of ectopic at our institution may reflect the burden of ectopic gestation in the society 
although it may not be used determine the regional incidence. It is more likely that the rate of ectopic pregnancies at the institution is more as it is a tertiary referral centre and hence the actual regional incidence may be lower than calculated. It is possible that some ectopic pregnancies may go undetected in rural areas as there may be low clinical suspicion observed at peripheral health centres and $25 \%$ of the cases presenting to our institution were in shock and this raises a suspicion if there was any maternal death before they could present to the hospital. Hence, it is likely that we are underestimating ectopic pregnancy as a cause of early maternal death and hence there is a need to estimate the overall contribution of ectopic pregnancy to maternal death. $^{7}$

The diagnosis of ectopic pregnancy in our study was predominantly based on a combination of history, clinical signs and ultrasound examination. Presence of complex adnexal mass was the most common ultrasound feature seen in more than $50 \%$ of the cases. Culdocentesis to aid diagnosis was used only in 5 cases and it was positive in 3 cases. Serum beta hCG was used to aid diagnosis in 11 cases and it was used to differentiate an ectopic pregnancy from failing intrauterine pregnancy. Diagnostic laparoscopy was gold standard in confirming ectopic pregnancy as in 3 cases where clinical and ultrasound suspicion of ectopic gestation was high, a subsequent laparoscopic examination revealed a corpus luteal cyst hemorrhage in 1 case and no evidence of ectopic gestation in other 2 cases (which were subsequently found to have failing intrauterine pregnancy). Diagnostic delays are commonly encountered in peripheral centres leading to women presenting with hemodynamic compromise and shock. This study emphasizes the importance of increasing awareness about the varied clinical presentations of ectopic pregnancy and the need to have a high index of clinical suspicion in women presenting with abnormal uterine bleeding in women of reproductive age group. Health workers in the peripheral set-up need to be educated about the various clinical presentations and also the various diagnostic modalities available to improve the diagnosis of ectopic pregnancy and should be urged to refer the women with suspected ectopic to tertiary centres at the earliest so that timely intervention prevents both morbidity and mortality to the patient. ${ }^{8-10}$

Presence of predisposing factors were evaluated through the details provided in the case sheet. Evaluation of the risk factors is subjected to documentation bias. The most common risk factors associated with ectopic pregnancy were STI's and subfertility which was documented in $34.8 \%$ and $30.23 \%$ of the cases respectively. Although PID was clinically demonstrated in 5 cases, intra-op evaluation revealed the presence of hydrosalphinx, tubercles and adhesions in 9 cases suggesting the role of subclinical infection causing tubal damage. Early sexual debut and lower rate of usage of contraception has led to higher incidence of STI's as documented in other settings. ${ }^{11-13}$ Usage of MTP kit by 2 women suggests that ultrasound confirmation of location of pregnancy prior to prescribing MTP kit is mandatory.

Salpingectomy was done in all cases with tubal ectopic either by open method or by laparoscopy. Ovarian ectopic were diagnosed after they fit into strict Speigelberg criteria. Oophorectomy was done in all 3 cases of ovarian ectopics. Women undergoing laparoscopic procedure had an earlier post-operative recovery and were discharged earlier from the hospital. Intra-peritoneal bleeding was present in more than $75 \%$ of the cases and majority of them with significant bleed required intra-op and post-op blood transfusion. Simultaneous resuscitation of the patient and laparotomy revived the women in shock sooner. Similar findings were documented in other studies supporting the fact that laparotomy was the operation of choice in women presenting with shock. ${ }^{14-17}$ Hence it is to be advocated to health personnel referring cases from distant rural areas to start fluid resuscitation prior to referral. Hormonal contraception for a minimum of six months was recommended. Approximately two-thirds of the women received blood transfusion and only 5 women received injectable iron to correct anaemia. Hence, hematinic supplementation was recommended for most of the women at the time of discharge.

There are certain areas which need further research. Firstly, the institutional rate of ectopic pregnancy calculated may not be projected on to the regional population. To determine the regional rate of ectopic gestation, a larger cross-sectional study involving different health centres helps in determining the regional rate of ectopic pregnancy. Secondly, the need to evaluate the sensitivity and specificity of available diagnostic modalities (USG, serum betas-hCG, culdocentesis) as we had 3 negative laparotomies. Lastly, a survey on knowledge and awareness among health workers may address the need for further training.

There are certain limitations of the study. First, the institutional rate approximately calculated for the year 2017 (only including first 4 months) may overestimate the rate and hence the need to re-estimate the rate of ectopic at the end of the year. Second, as the entire study is based on the case sheet notes, there is a possibility of documentation bias. Lastly, although there was no maternal mortality in our study, it is likely that ectopic pregnancy causing maternal death might have been missed and such cases might have not reached the hospital.

\section{CONCLUSION}

Ectopic pregnancy is a common gynaecological emergency at tertiary hospital set-up. With rising incidence, a high index of clinical suspicion along with appropriate usage of diagnostic aids helps in timely detection and management of women with ectopic 
pregnancy. STI's and subfertility are the most prevalent risk factors. Hence interventions to prevent and treat STI's are required. Increasing the knowledge of the health workers regarding the ectopic pregnancy and by training and equipping them with diagnostic facilities help them to refer women with suspected ectopic to a tertiary hospital at the earliest to reduce both maternal morbidity and mortality.

\section{ACKNOWLEDGMENTS}

Authors would like to acknowledge the assistance and the technical support by the medical records department of SSIMS and RC, Davangere.

Funding: No funding sources Conflict of interest: None declared

Ethical approval: Not required

\section{REFERENCES}

1. Faraquhar CM. Ectopic pregnancy. Lancet. 2005;366(9485):583-91.

2. Sivalingam VN, Duncan WC, Kirk E, Shephard LA, Horne AW. Diagnosis and management of ectopic pregnancy. J Fam Plann Reprod Health Care. 2011;37(4):231-40.

3. Jurkovic D, Wilkinson $\mathrm{H}$ : Diagnosis and management of ectopic pregnancy. BMJ. 2011;342:d3397.

4. Anrolu RI, Oluwole A, Abudu OO, Adebajo S: Risk factors for ectopic pregnancy in Lagos, Nigeria. Acta Obstet Gynecol Scand. 2005;84(2):184-8.

5. Indian Council of Medical Research Task Force Project: Multicentre case-control study of ectopic pregnancy in India. J Gynaecol Obstet India. 1990;40:425-30.

6. Eshan N, Mehmood A. Ectopic pregnancy: an analysis of 62 cases. J Pak Med Assoc. 1998;48(2):26-9.

7. de Rosany P, Irvine LM. Reporting rates of ectopic pregnancy: are we any closer to achieving concensus? J Obstet Gynaecol. 2012;32(1):64-7.
8. Bag TS, Saha DP, Dasgupta N, Sarkar M, Mandal SK, Mondal $\mathrm{T}$ et al. Time trends in ectopic pregnancy over a decade- a retrospective hospital based study. J Indian Med Assoc. 2012;109(10):7279.

9. De Muylder X. Ectopic pregnancy in Zimbabwe. Int J Gynecol Obstet. 1991;35(1):55-60.

10. Wedderburn CJ, Warner P, Graham B, Duncan WC, Critchely HO, Horne AW. Economic evaluation of diagnosing and excluding ectopic pregnancy. Hum Reprod. 2009;25(2):328-33.

11. Ville Y, Leruez M, Glowaczower E, Robertson JN, Ward ME. The role of Chlamydia trachomatis and Neisseria gonorrhoeae in the aetiology of ectopic pregnancy in Gabon. $\mathrm{Br} \mathbf{J}$ Obstet Gynaecol. 1991;98(12):1260-6.

12. Lindow SW, Moore PJ. Ectopic pregnancy: analysis of 100 cases. Int J Gynaecol Obstet. 1988;27(3):3715.

13. Bugalho A, Strolego F, Pregazzi R, Osman N, Ching C. Extrauterine pregnancy In Mozambique. Int $\mathbf{J}$ Gynaecol Obstet. 1991;34(3):239-42.

14. Amoko DH, Buga GA. Clinical presentation of ectopic pregnancy in Transkei, South Africa. East Afr Med J. 1955;72(12):770-3.

15. Gharoro EP, Igbafe AA. Ectopic pregnancy revisited in Benin City,Nigeria: analysis of 152 cases. Acta Obstet Gynaecol Scand. 2002;81(12):1139-43.

16. Bouyer J, Coste J, Shojaei T, Pouly JL, Fernandez H, Gerbaud L et al. Risk factors for ectopic pregnancy: a comprehensive analysis based on a large casecontrol, population-based study in France. Am J Epidemiol. 2003;157(3):185-94.

17. Kouam L, Kamdom-Moyo J, Doh AS, Ngassa P. Treatment of ectopic pregnancies by laparotomy in under-equipped countries. A series of 144 cases at the younde university hospital center (Cameroon). J Gynecol Obstet Biol Reprod (Paris). 1996,25(8):8048.

Cite this article as: Malavika JC, Prabhudev P, Bandamma NS. A clinical study of ectopic pregnancy: a five-year institutional experience. Int $\mathbf{J}$ Reprod Contracept Obstet Gynecol 2017;6:2168-73. 